\title{
Evaluation of Exam Anxiety in Healthy Subjects using Brain Signals Analysis
}

\author{
Parisa Ghafourian ${ }^{1}$, Majid Ghoshuni ${ }^{*}$, Iraj Vosoogh ${ }^{2}$ \\ ${ }^{1}$ Department of Biomedical Engineering, Mashhad Branch, Islamic Azad University, Mashhad, Iran \\ ${ }^{2}$ Mashhad University of Medical Sciences, Mashhad, Iran
}

\section{ABSTRACT}

Introduction: Recent studies have proven that anxiety disorders have the highest abundance throughout the world. Almost everyone has experienced an anxiety. This anxiety can have an agonizing impact on a person's life; however, anxiety can also be invigorating. Invigorating anxiety pushes a person to work with a goal in mind, while the more detrimental type of anxiety limits his or her attention. The purpose of this study was to investigate the brain function in test anxiety during answering mathematical questions. Material and Methods: In this experiment, 22 participants ( 9 male and 13 female) divided in two groups of test and control. Speelberger and ASRS anxiety tests were taken from all of the participants. Then electroencephalogram signal was recorded on 19 channels for 5 minutes with their eyes open and they were taking a conceptual math test simultaneously. In comparison with the control group, the test group had a shorter time to answer the questions and the video of the testgroup subjects was recorded while answering to a serious tester. After signal preprocessing, using Neuroguide software, frequency band powers of brain signal was extracted and the inattentional index (Theta/Beta ratio) was compared between the test and control groups using paired sample t-test. Results: In the test group, a significant decrease in theta to beta index was observed during math test compared to the eyes open condition on T3 $(p=0.077, t=1.96), T 4(p=0.026$, $\mathrm{t}=2.619)$, T5 $(\mathrm{p}=0.084, \mathrm{t}=1.91)$ channels. Besides, a nearly significant correlation $(\mathrm{r}=0.4055$, $\mathrm{p}=0.0612$ ) was found between false answers and percent change of theta/beta index during math test compared to rest condition. Conclusion: Anxiety in the test group was invigorating and reduced test error and inattention index. In the healthy subjects, due to the imposing anxiety from tester, the level of attention of the subjects increased significantly and their error in answering the questions decreased.

\section{Key words:}

1. Performance Anxiety

2. Electroencephalography

3. Psychological Phenomena

*Corresponding Author: Majid Ghoshuni

E-mail: ghoshuni@mshdiau.ac.ir 
ارزيابى اضطراب آزمون در افراد سالم با استفاده از تحليل سيگنال مغزى

\author{
"يريسا غفوريان'، مجيد قشونى "'، ايرج وثوق' \\ اكروه مهيندسى يزشكى، واحد مشهيد، دانشخاه آزاد اسلامى، مشهر، ايران

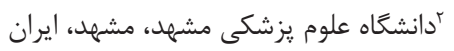

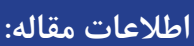

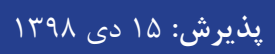

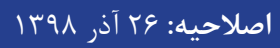

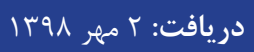

: بن : كليد وازهها:

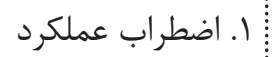

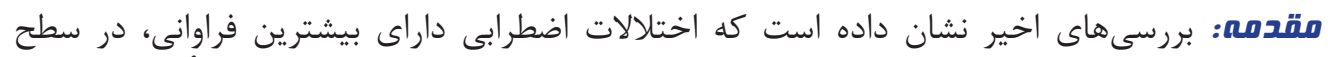

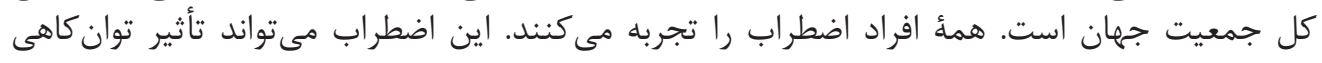

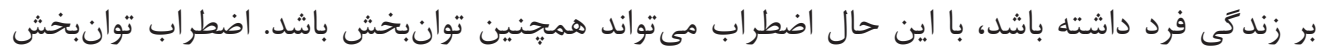

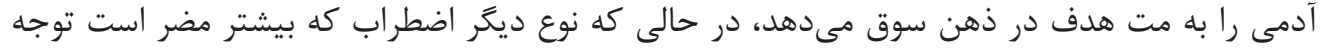

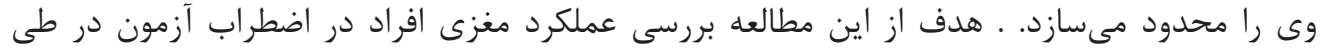

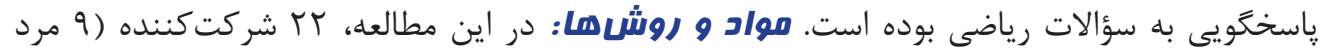

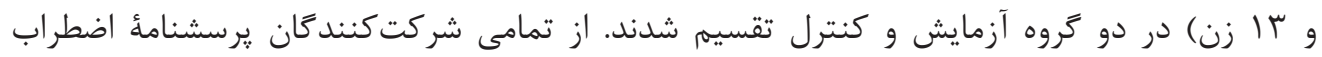

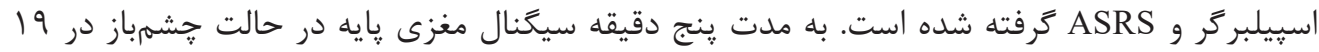

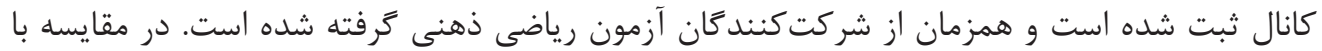

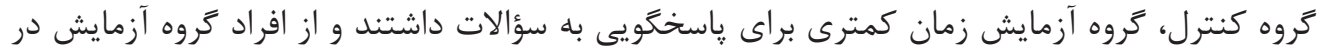

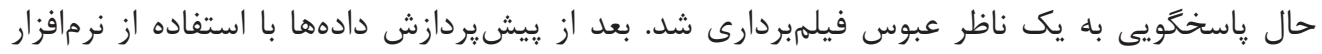

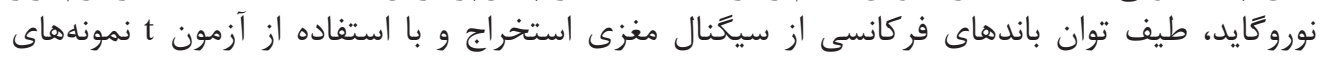

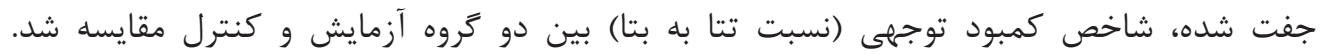

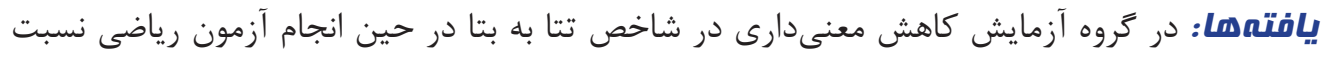

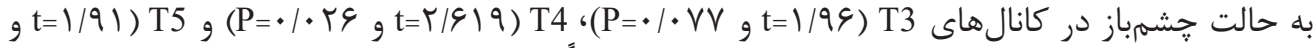

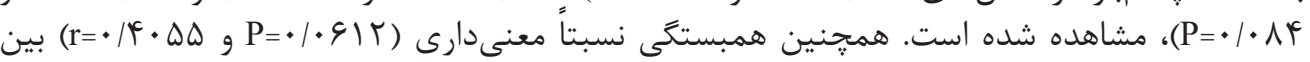

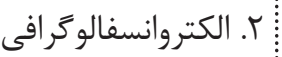

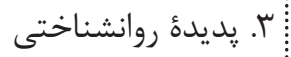

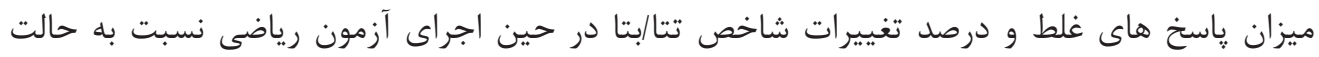

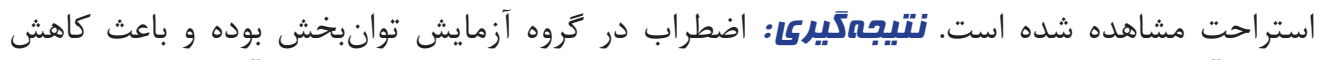

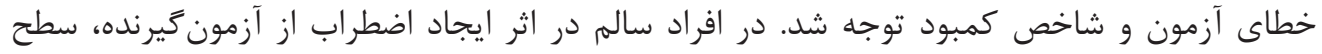

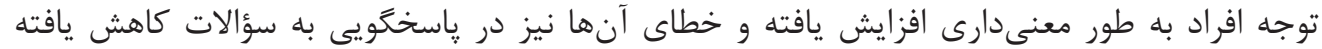




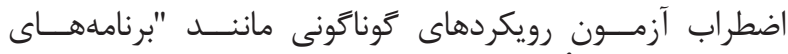

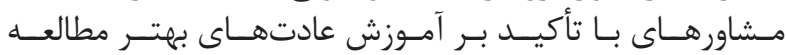

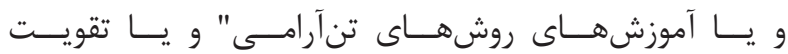

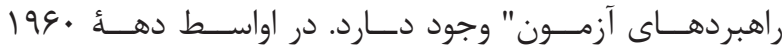

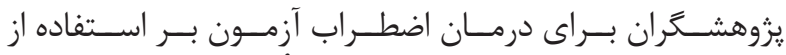

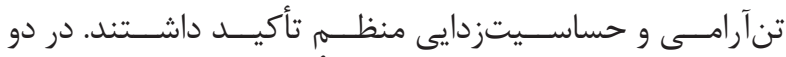

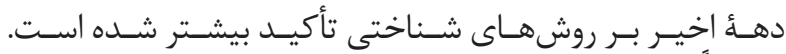

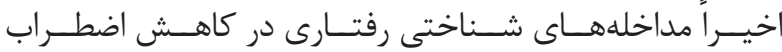

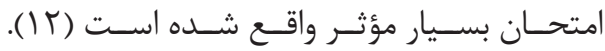

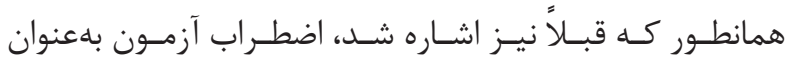

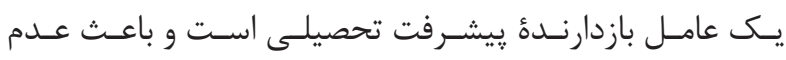

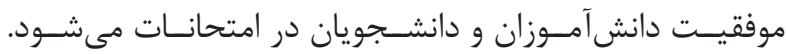

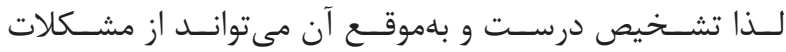

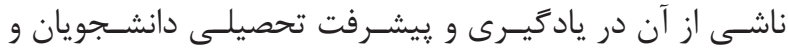

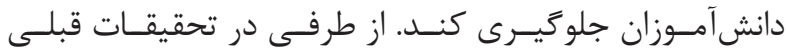

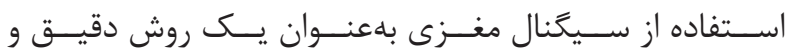

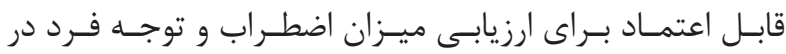

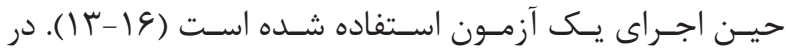

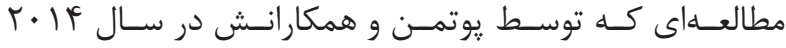

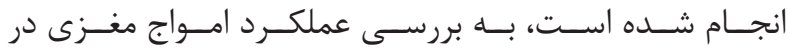

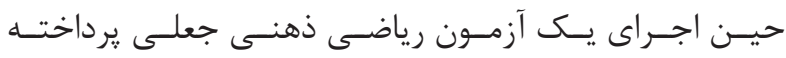

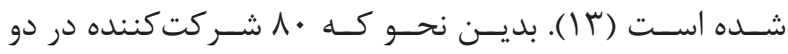

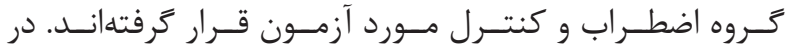

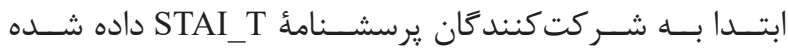

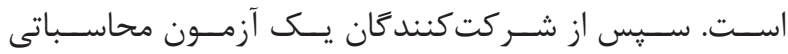

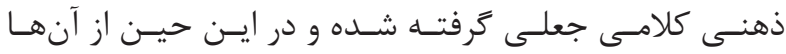

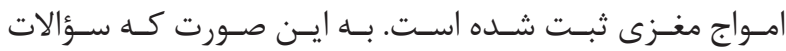

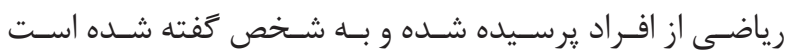

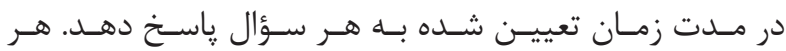

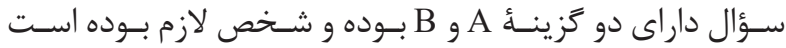

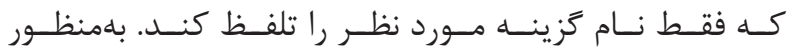

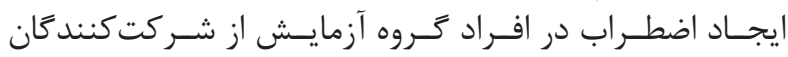

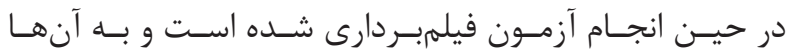

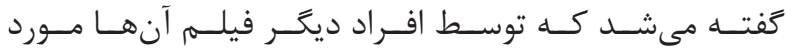

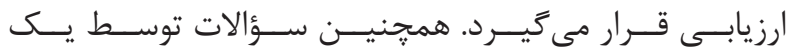

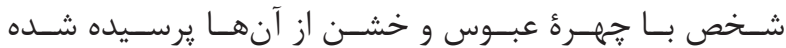

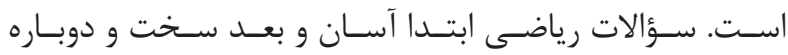

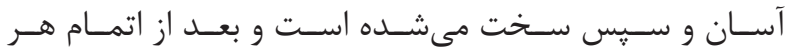

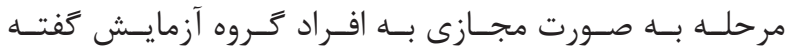

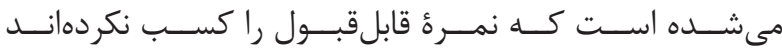

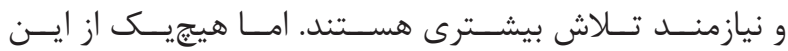

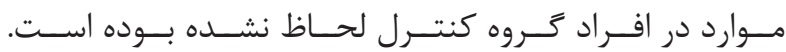

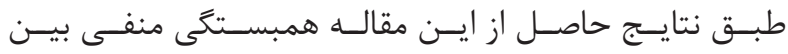

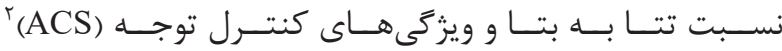

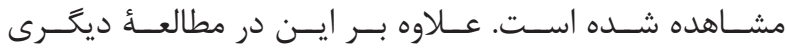

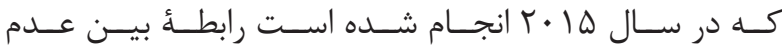

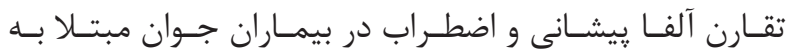

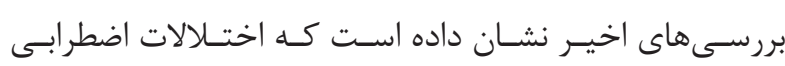

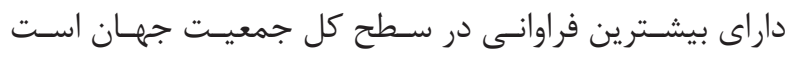

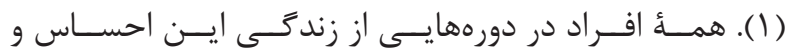

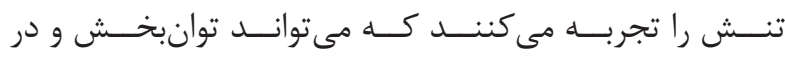

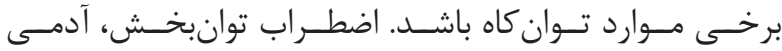

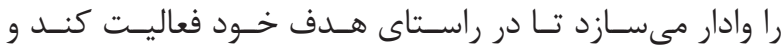

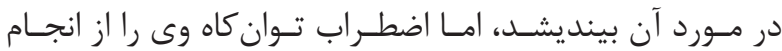

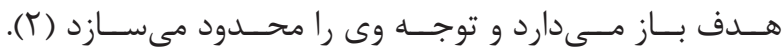

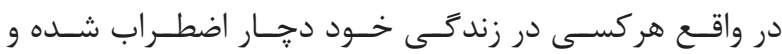

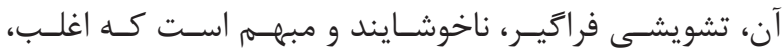

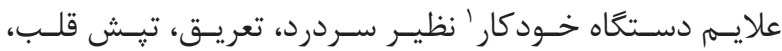

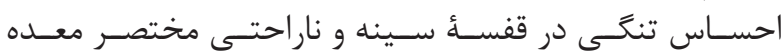

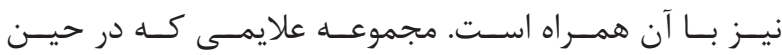

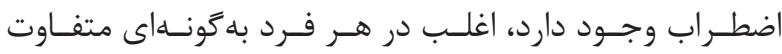

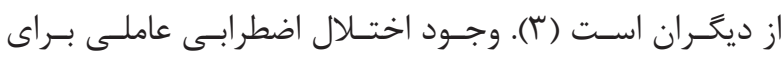

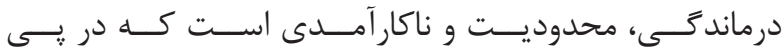

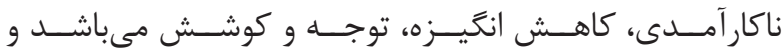

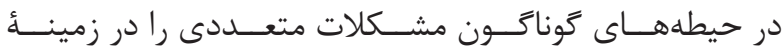

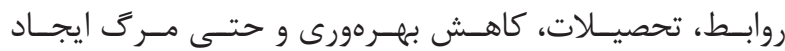

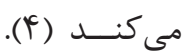

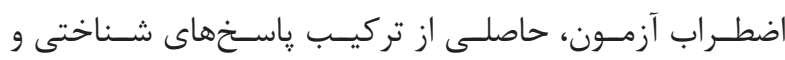

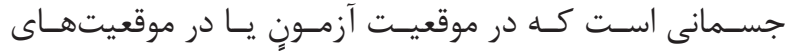

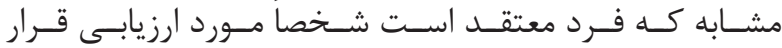

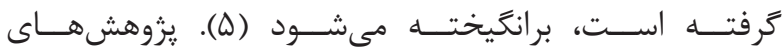

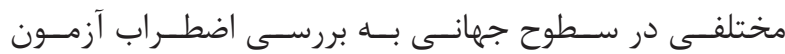

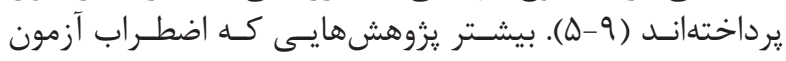

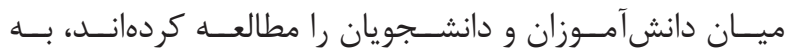

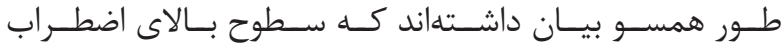

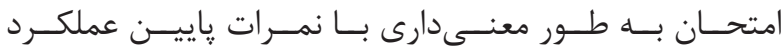

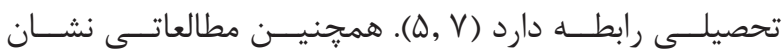

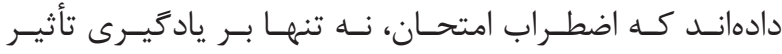

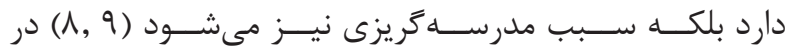

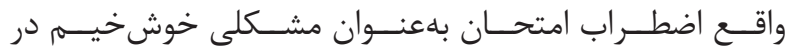

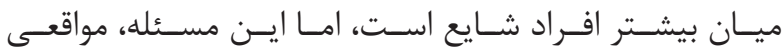

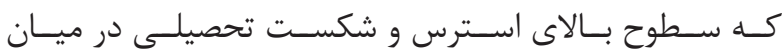

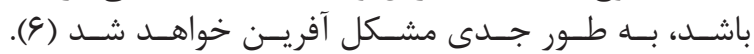

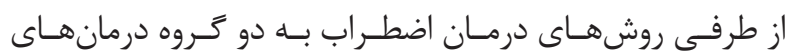

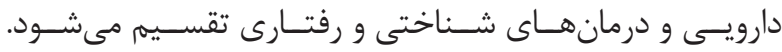

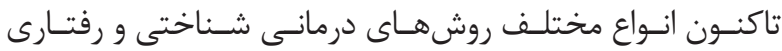

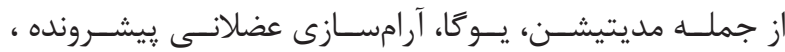

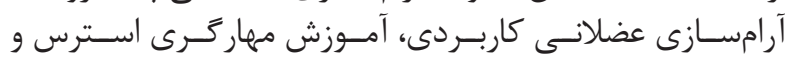

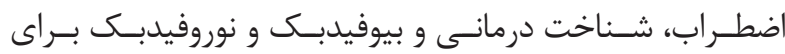

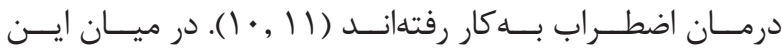

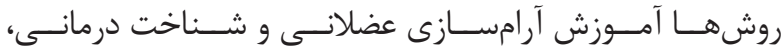

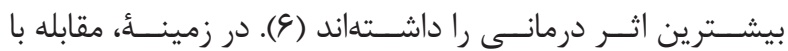




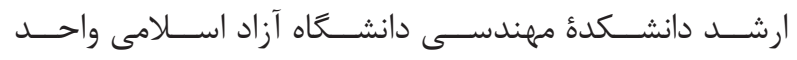

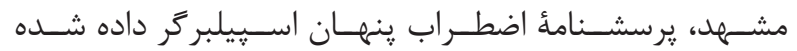

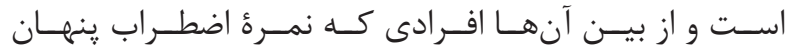

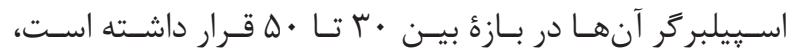

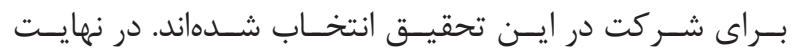

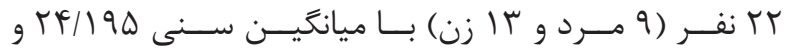

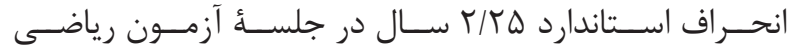

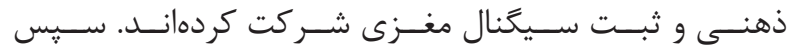

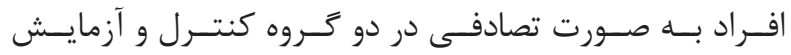

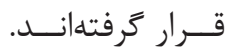
نحوهُ ثبت سيحنال مغزى

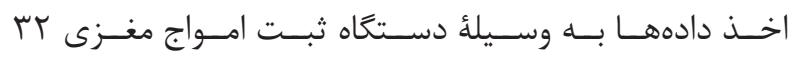

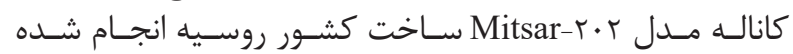

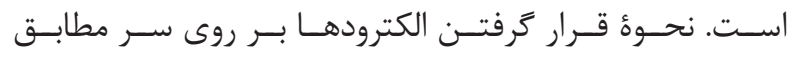

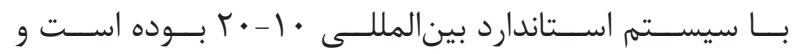

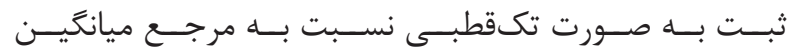

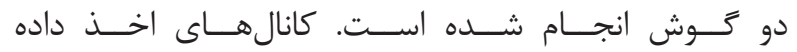

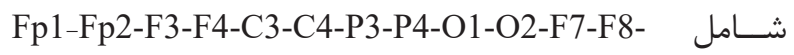
T3-T4-T5-T6-Fz-Pz-Cz اســت. در ايــن تحقيـق از كلاه

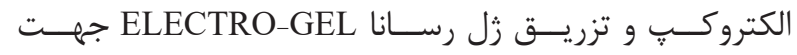

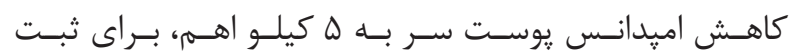

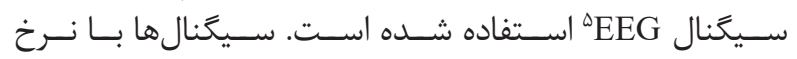

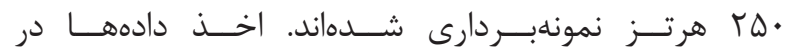

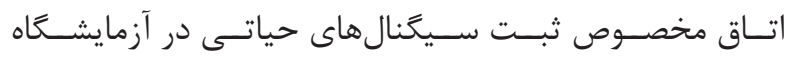

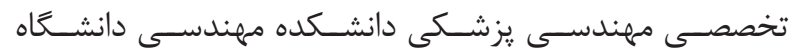

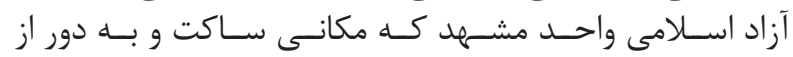

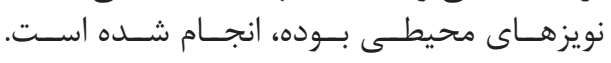

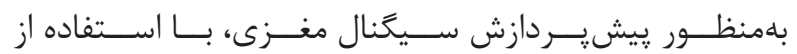

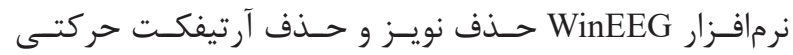

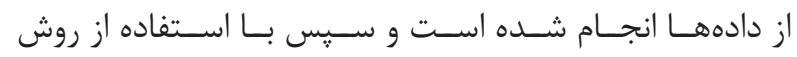

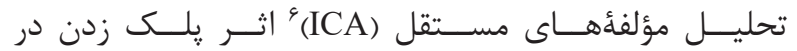

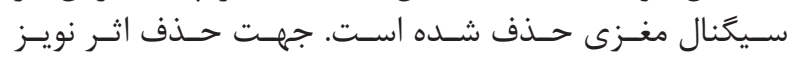

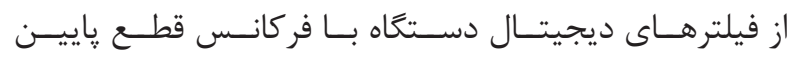

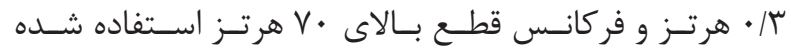

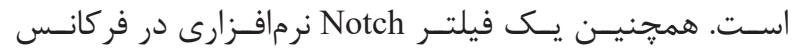
است

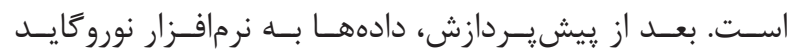

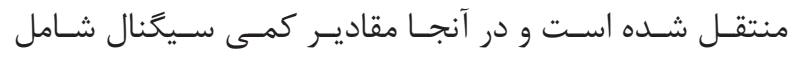

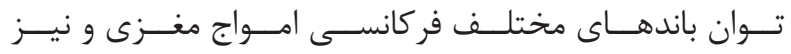

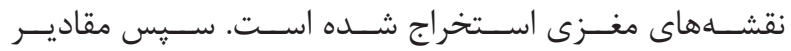

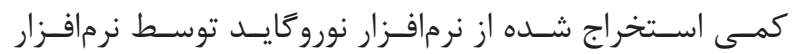

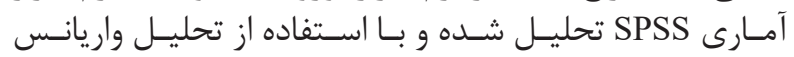

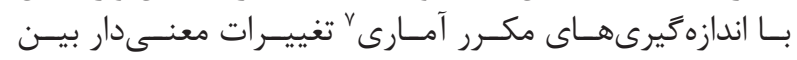

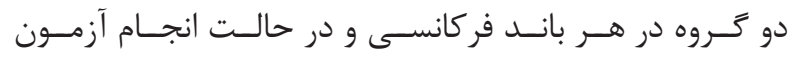

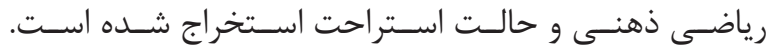

${ }^{3}$ Visual continuous performance task

${ }^{4}$ Auditory continuous performance task

${ }^{5}$ Electroencephalogram

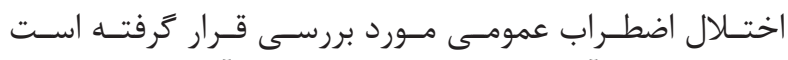

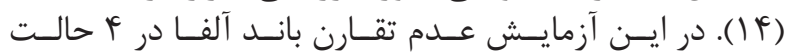

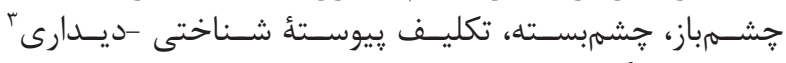

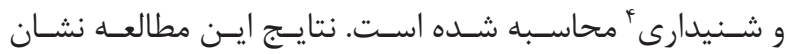

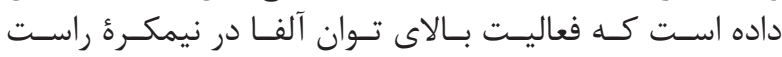

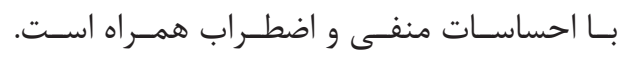

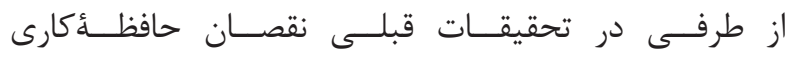

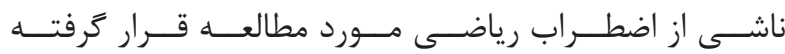

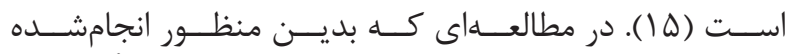

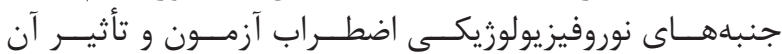

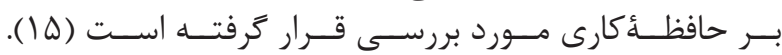

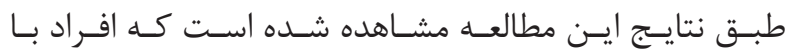

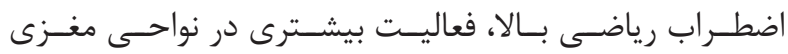

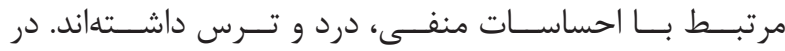

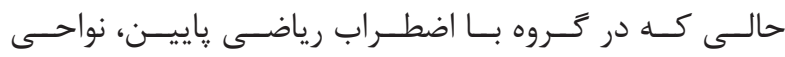

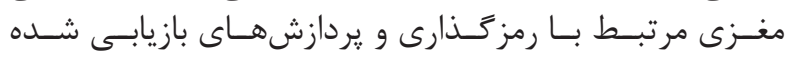

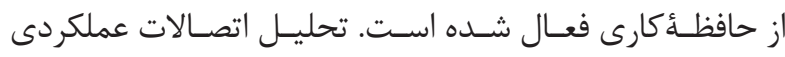

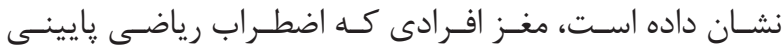

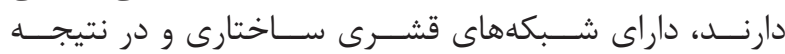

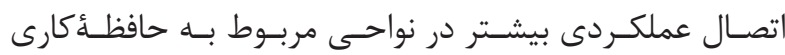

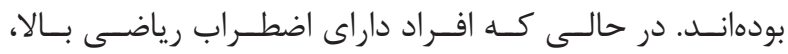

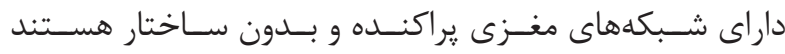

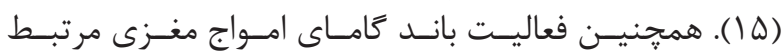

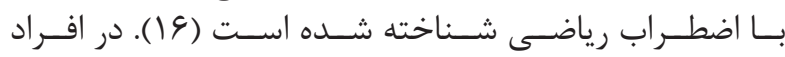

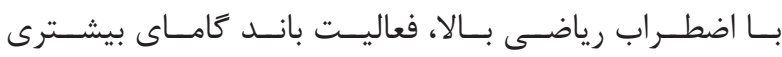

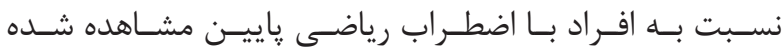

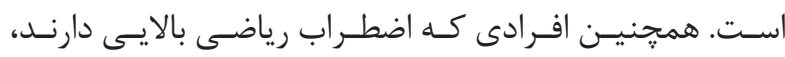

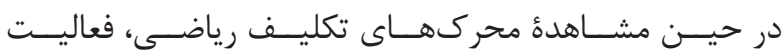

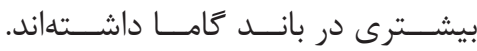

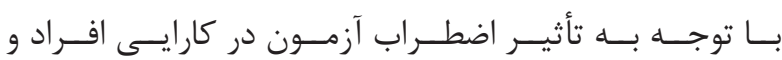

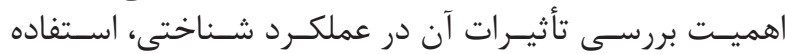

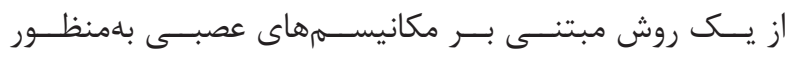

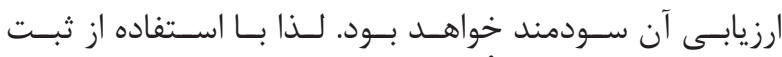

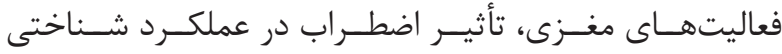

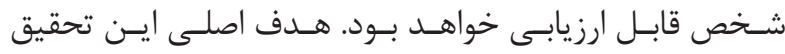

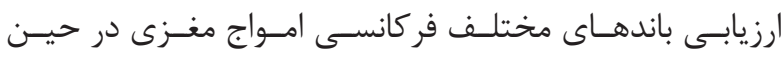

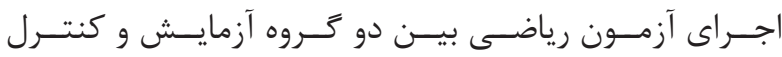

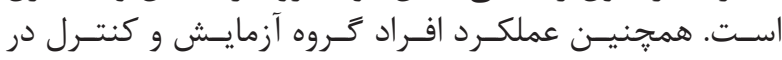

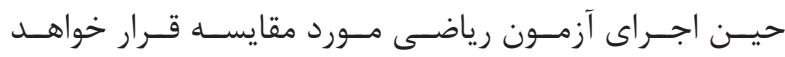
كرفت جنت

مواد و روشها مشخصات شر كت كنند

جهـت ثبـت دادههــاى مـورد نيـاز در ايسن تحقيـق، ابتـدا بـهـ

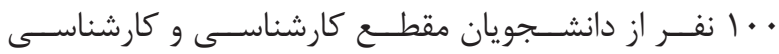

${ }^{6}$ Independent component analysis

${ }^{7}$ Repeated measure ANOVA 


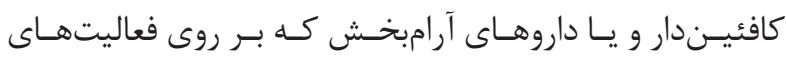

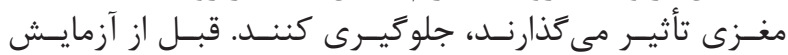

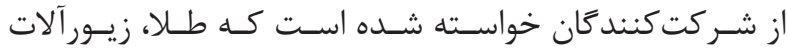

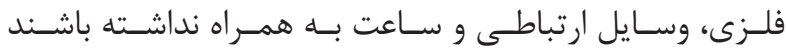

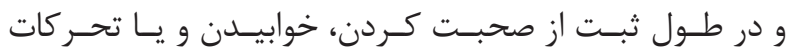

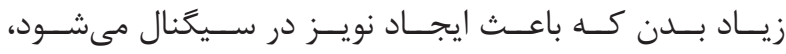

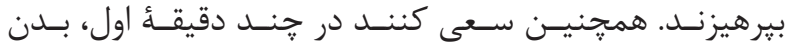

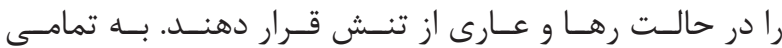

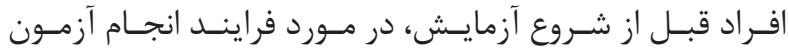

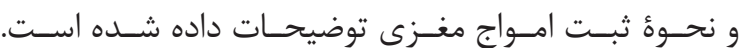

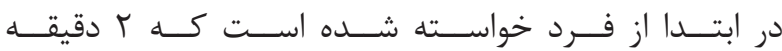

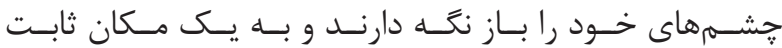

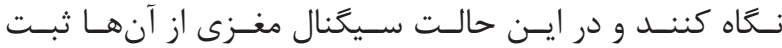

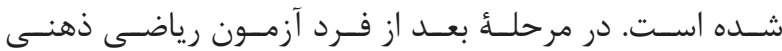

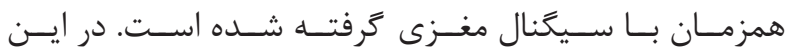

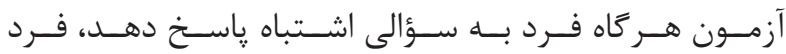

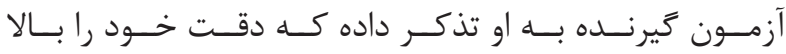

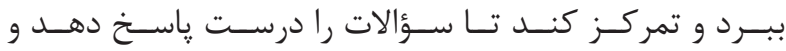

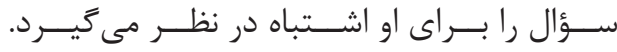

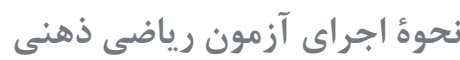

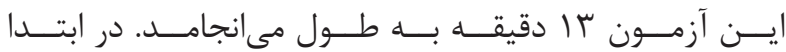

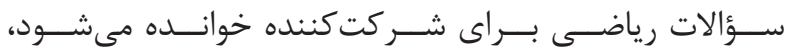

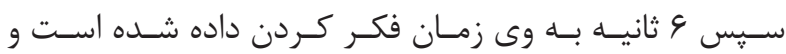

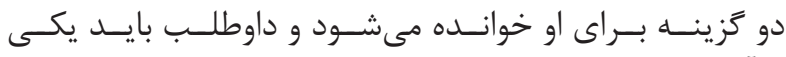

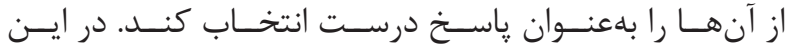

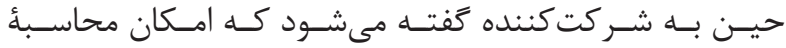

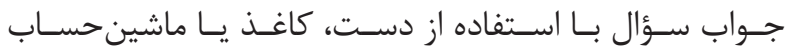

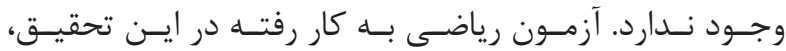

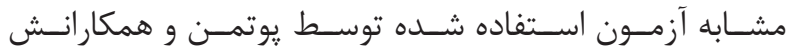

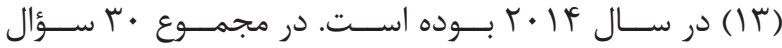

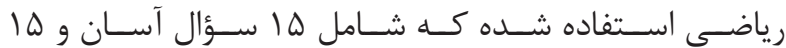

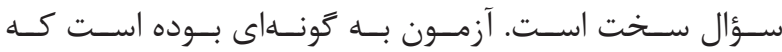

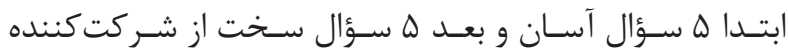

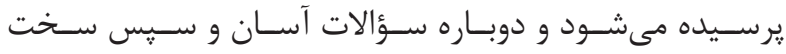

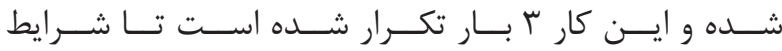

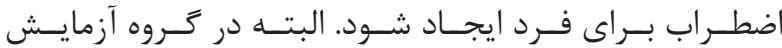

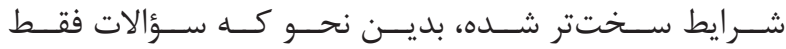

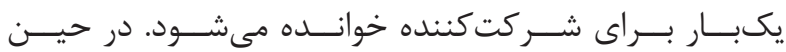

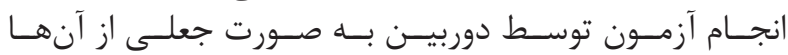

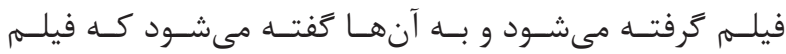

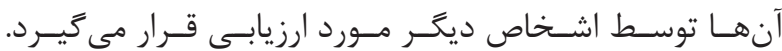

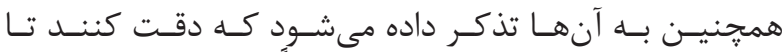

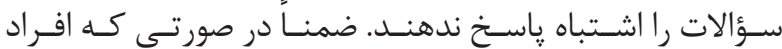

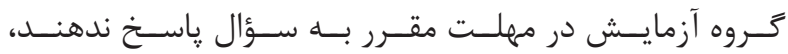

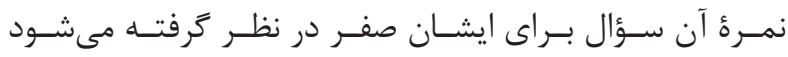

فر ايند اجراى آزمون

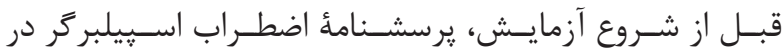

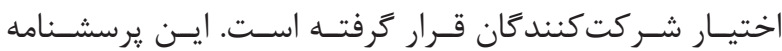

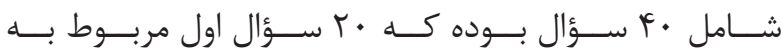

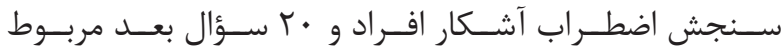

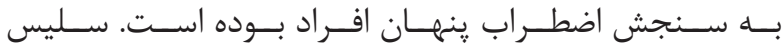

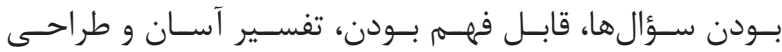

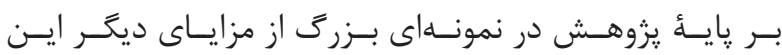

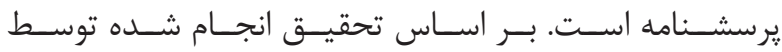

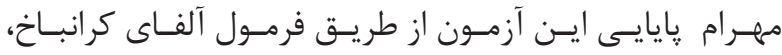

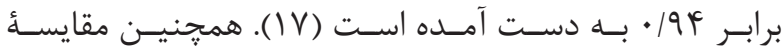

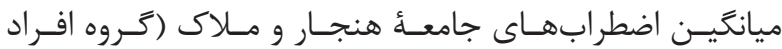

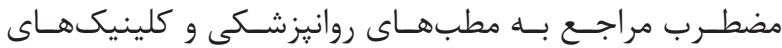

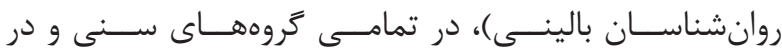

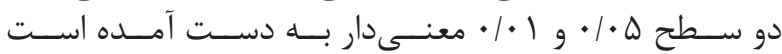

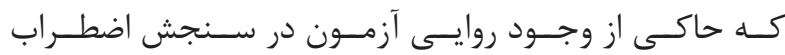

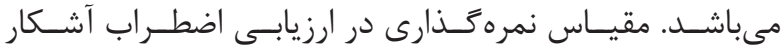

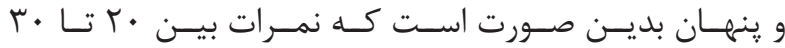

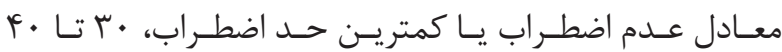

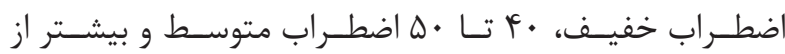

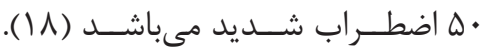

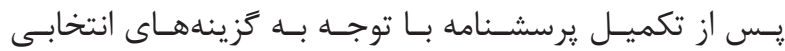

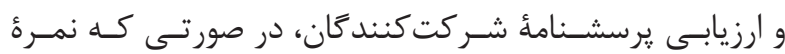

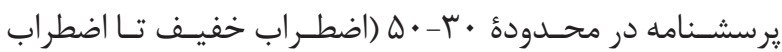

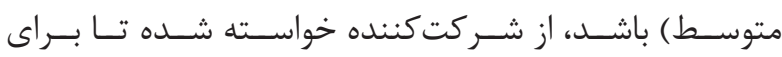

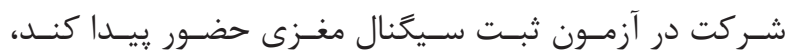

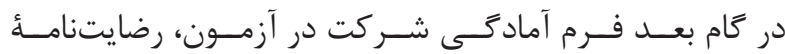

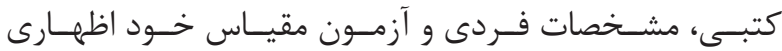

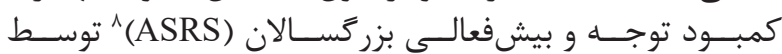

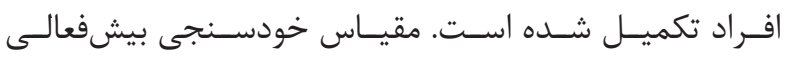

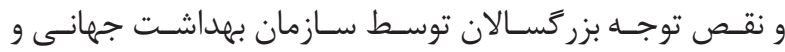

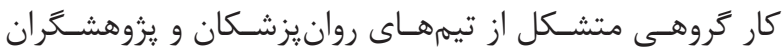

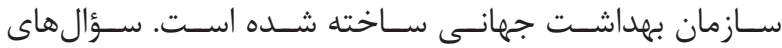

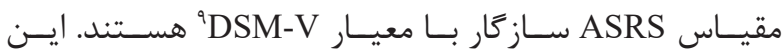

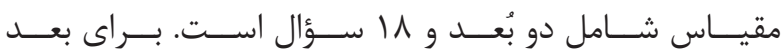

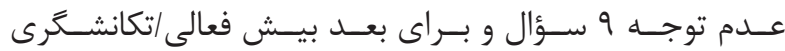

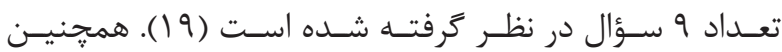

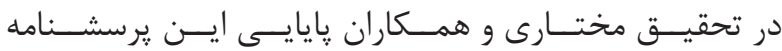

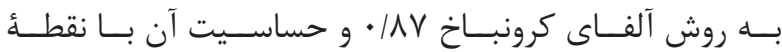

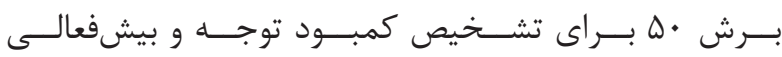

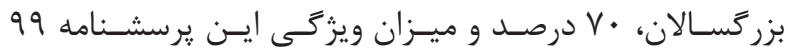

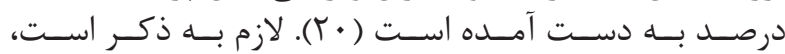

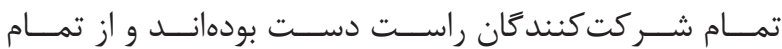

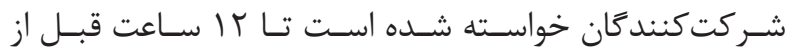

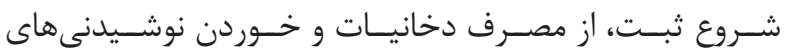




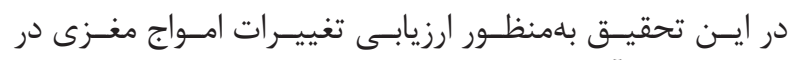

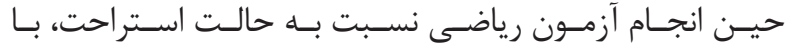

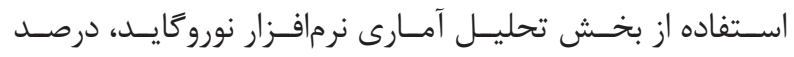

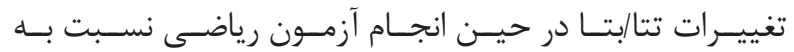

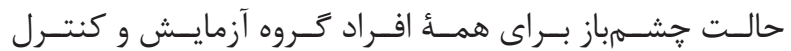

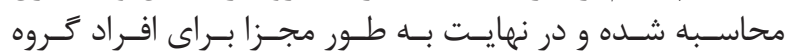

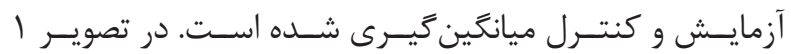

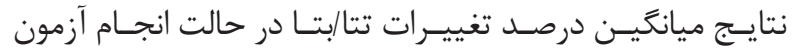

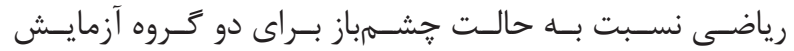

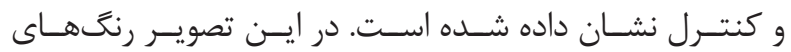

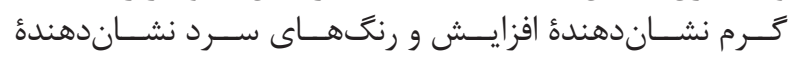

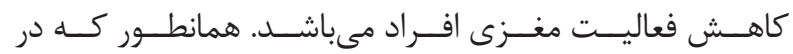

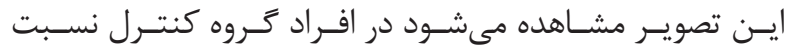

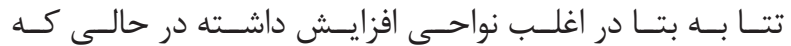

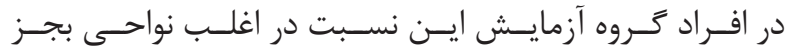

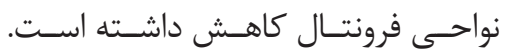

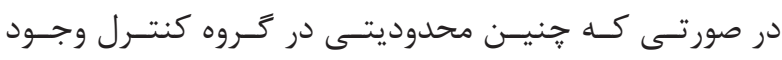

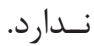

يافتهها

نتايج يرسشنامذ ASRS و اسيبلر

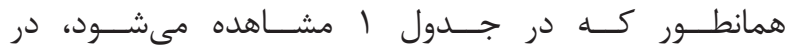

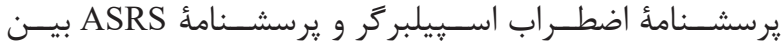

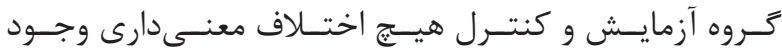

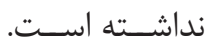
نتايج آزمون رياضى ذهنى

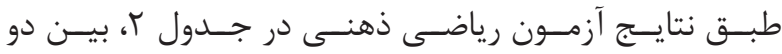

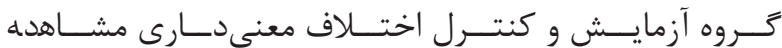
نشـده اســت نتايج تحليل امواج مغزى

جدول ا- نتايج يرسشنامئ اضطراب آشكار اسييلبرگر.

\begin{tabular}{|c|c|c|c|c|c|}
\hline سطح معثى دارى & أنحراف استاندارد & سطح اضطراب & ميانكين & og & \multirow{3}{*}{ نتايج يرسشثامةُ اضطراب آشكار استيلبركّر } \\
\hline \multirow{2}{*}{. $\mid \Lambda G G}$. & VIAVIV & ب خفيف & rV/Gr & 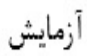 & \\
\hline & $V / \cdot V \& q$ & ب خفيف & $r q / \Lambda 1$ & كثترل & \\
\hline \multirow{2}{*}{ - /DVAr } & $q / V F q q$ & خ خفيف & TN/NI & 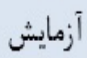 & \multirow{2}{*}{ نتايج يرسشتامة اضطراب بثهان اسيبلبرئر } \\
\hline & $N H \cdot F F$ & اب خفيف & $f+1 *$ & & \\
\hline \multirow{2}{*}{$+|\Delta| 9 \mid$} & $\psi / \cdot \Delta \wedge V$ & كمبود توجئ بسيار خفيف & $Y F / F D$ & 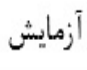 & \multirow{2}{*}{ تتابتج بِرسششاملة ASRS } \\
\hline & $V / \varphi \Delta q \psi$ & بدون اختلال & YY/VY & كثترل & \\
\hline
\end{tabular}

جدول r- - متايج آزمون رياضى ذهنى.

\begin{tabular}{|c|c|c|c|c|}
\hline معنى دارى & انحراف استاندارد & ميانگين & 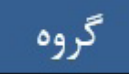 & \multirow{3}{*}{ نتايج آزمون رياضى ذهنى } \\
\hline \multirow{2}{*}{.1940} & $r / r r$ & 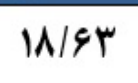 & آزمايش & \\
\hline & $r / \Delta$. & 19 & كنترل & \\
\hline
\end{tabular}
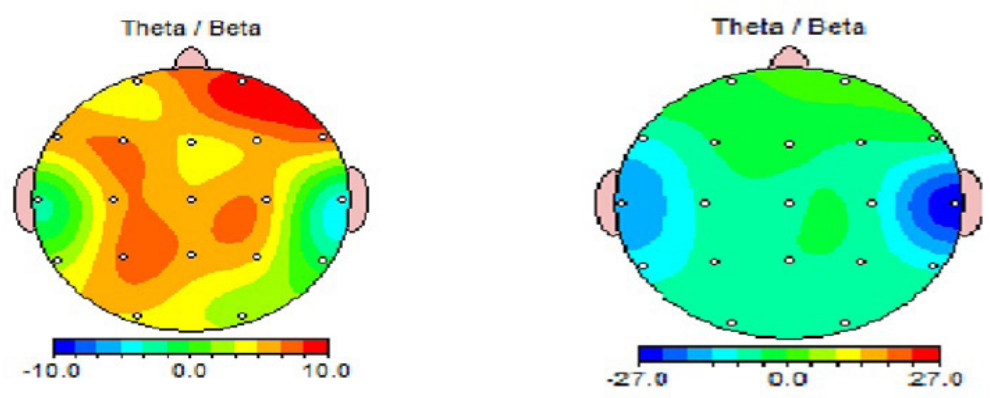

- ثمان

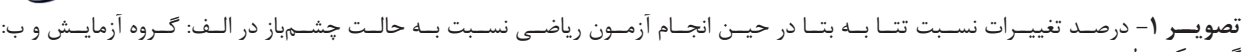




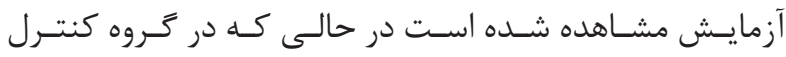

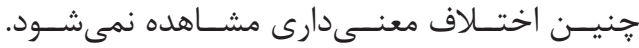

$$
\begin{aligned}
& \text { نتايج تحليل همبستخى }
\end{aligned}
$$

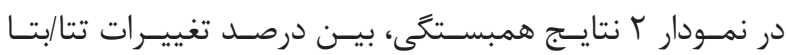

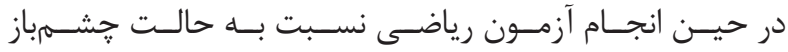

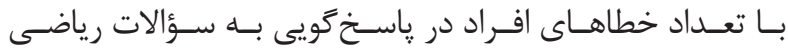

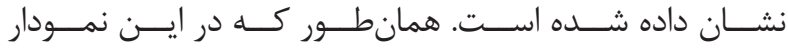

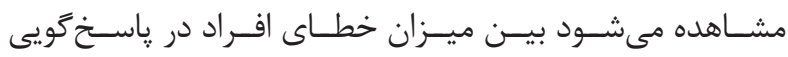

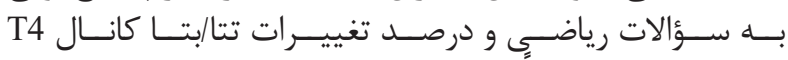

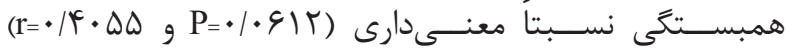

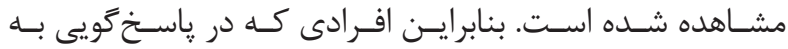

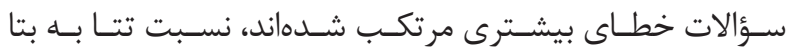

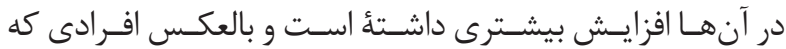

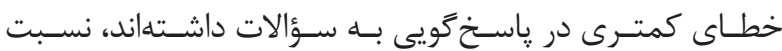

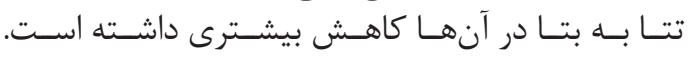
بحث و نتيجه
نتايج تحليل آمارى

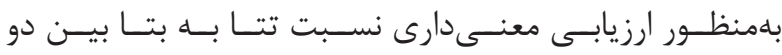

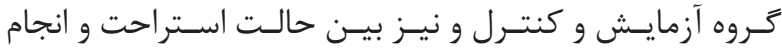

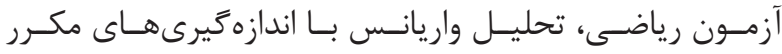

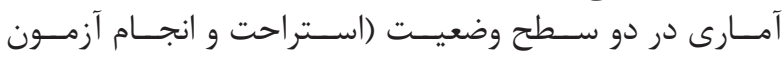

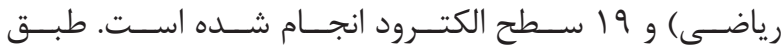

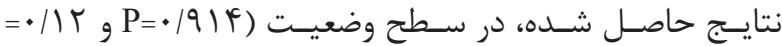

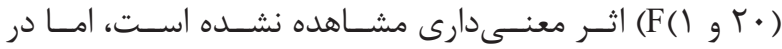

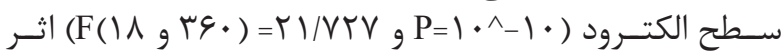

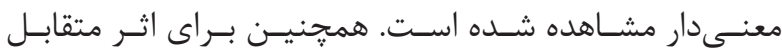

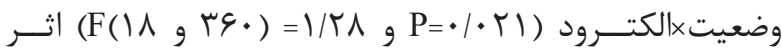

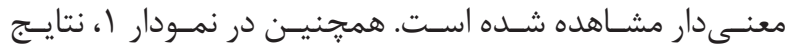

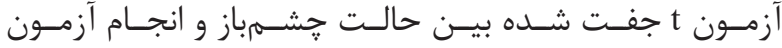

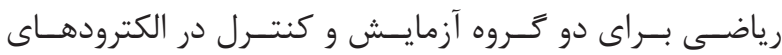

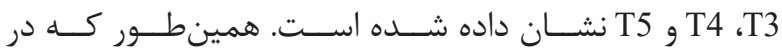

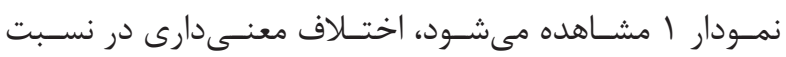

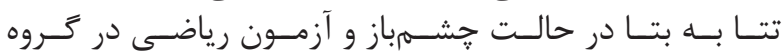
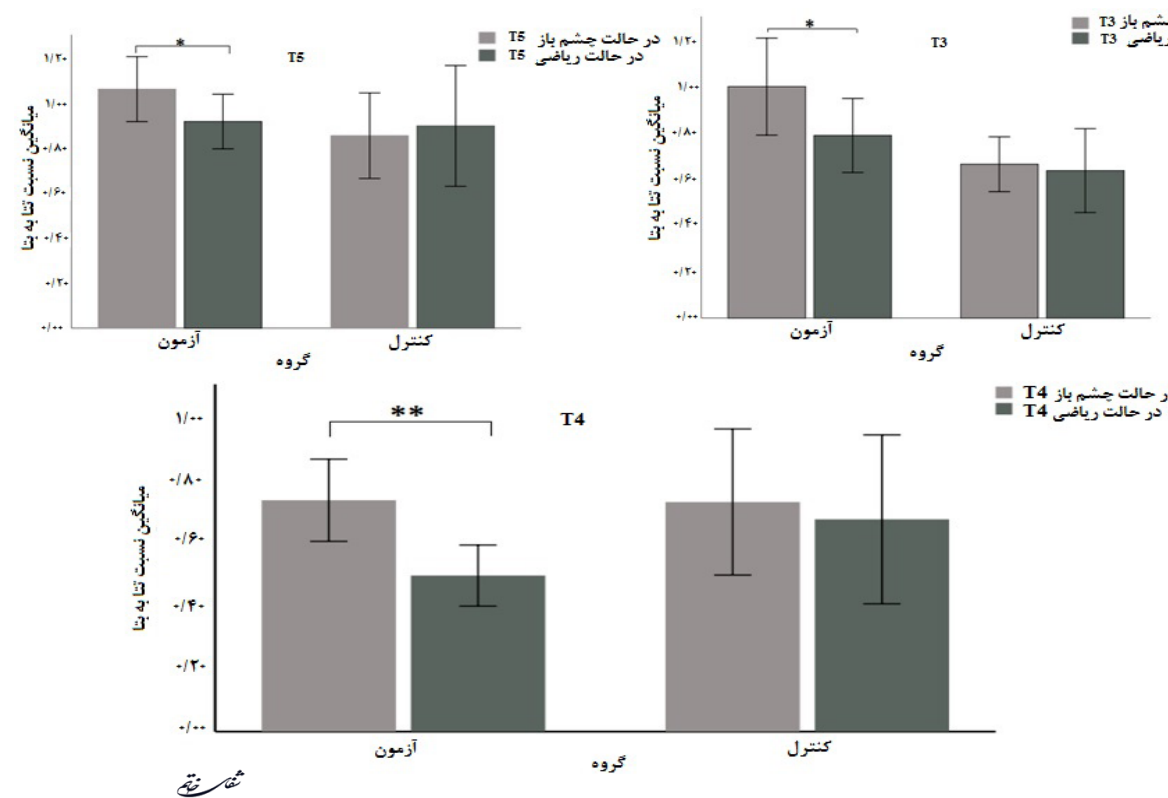

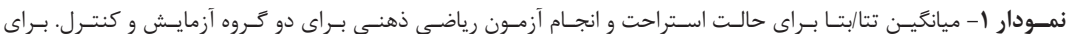

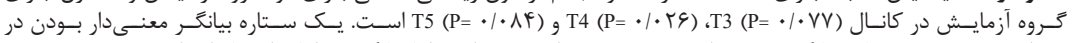

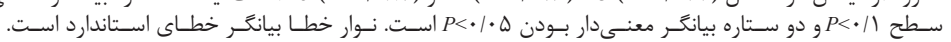

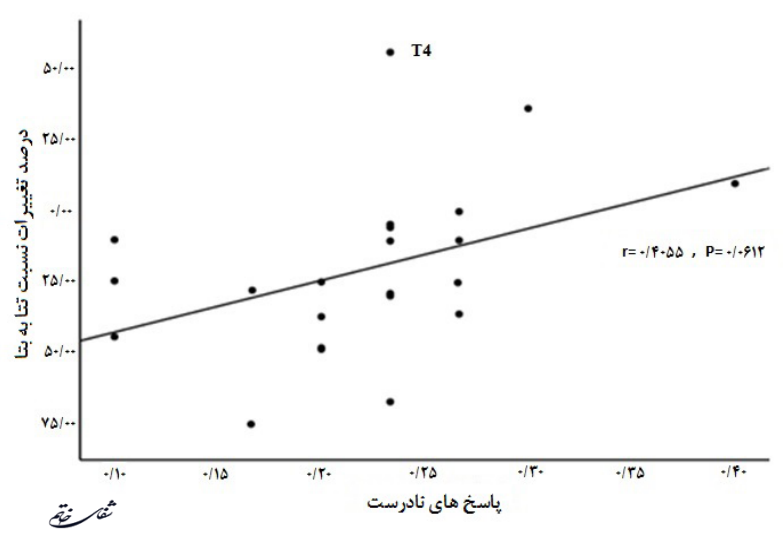

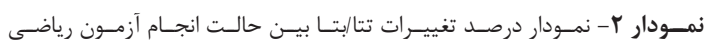

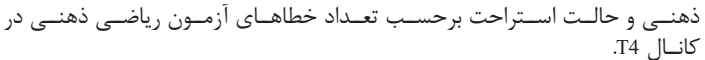




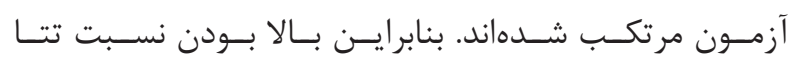

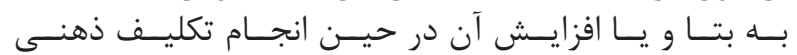

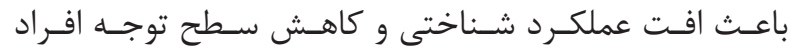

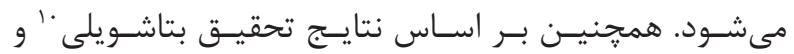

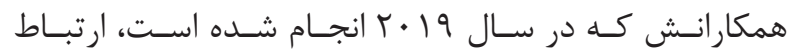

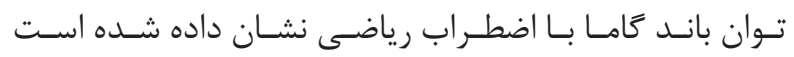

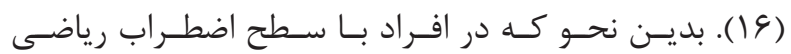

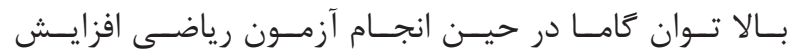

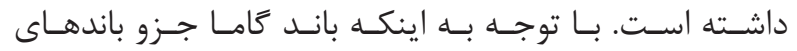

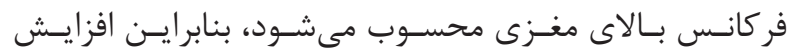

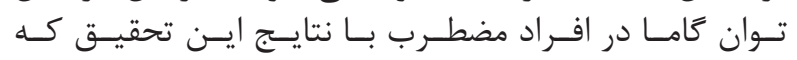

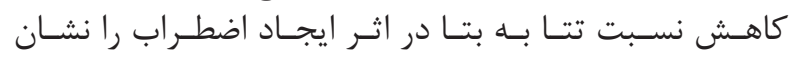

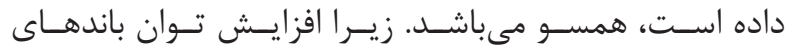

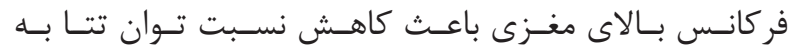

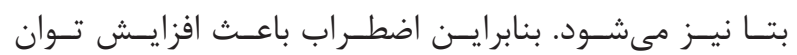

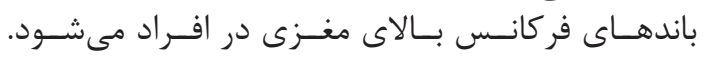

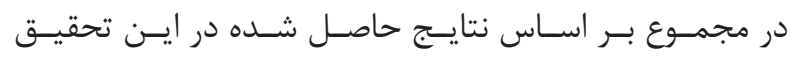

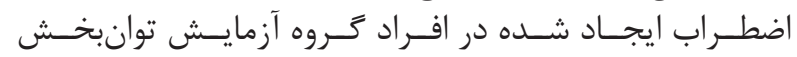

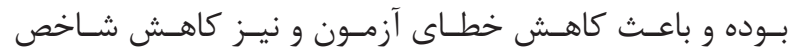

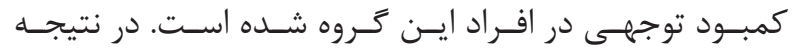

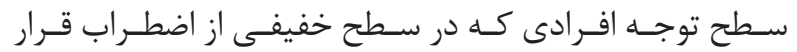

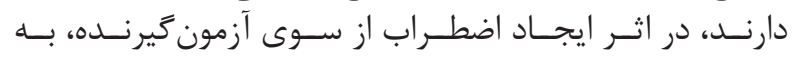

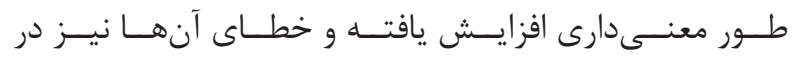

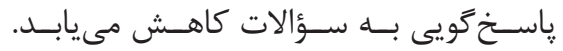

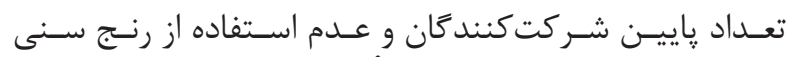

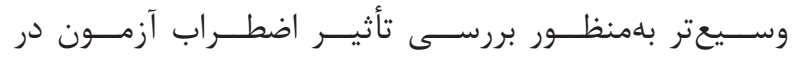

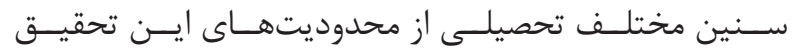

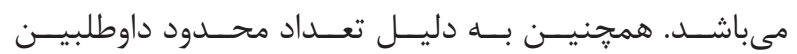

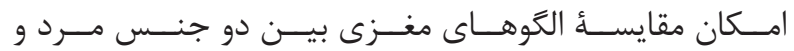

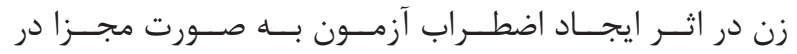

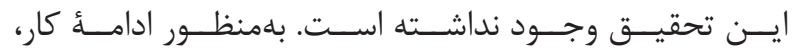

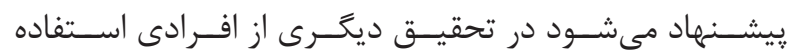

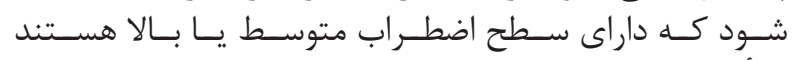

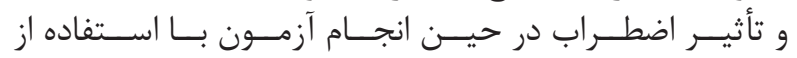

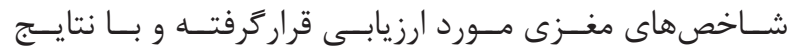

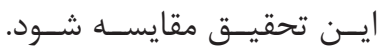

$$
\text { تشكر و قدردانى }
$$

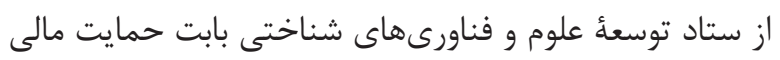
از اين تحقيق تشكر و قدردانى مى فئود

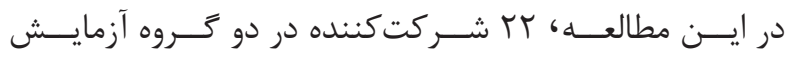

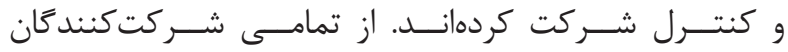

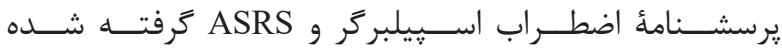

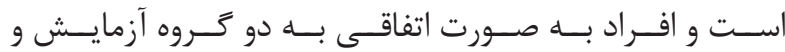

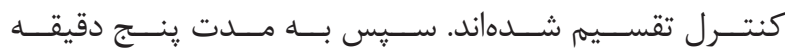

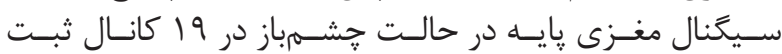

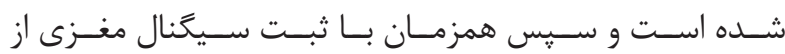

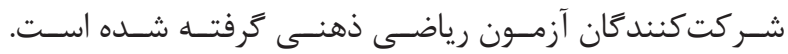

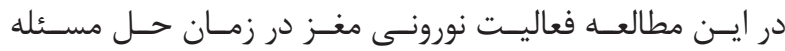

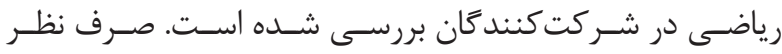

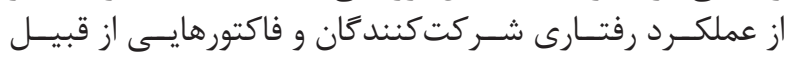

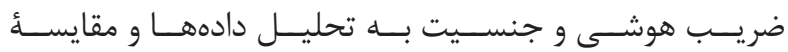

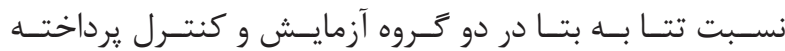

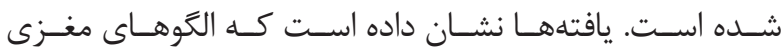

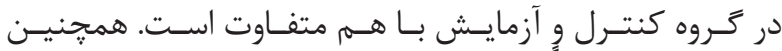

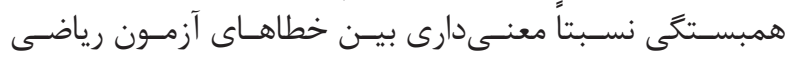

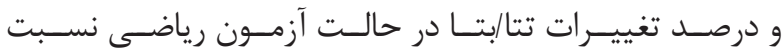

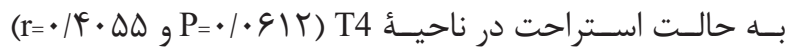
مشـاهده شــده اسـتـت

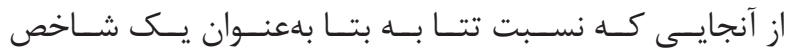

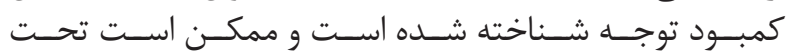

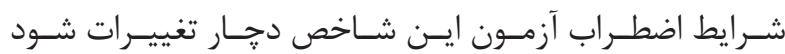

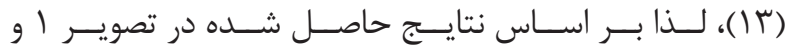

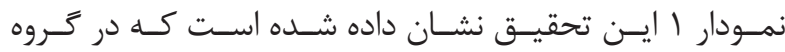

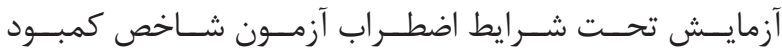

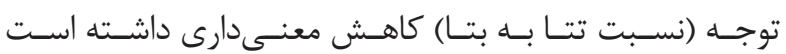

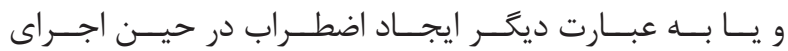

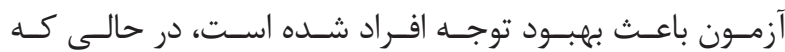

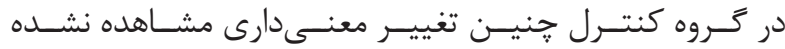

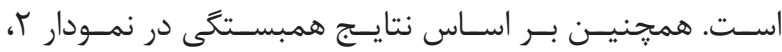

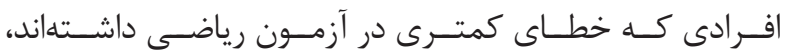

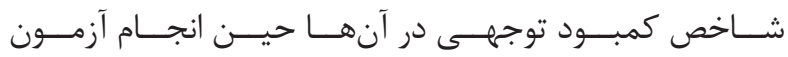

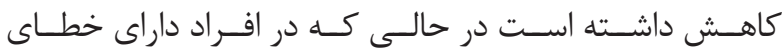

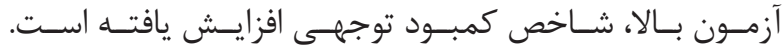

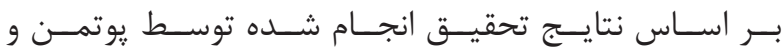

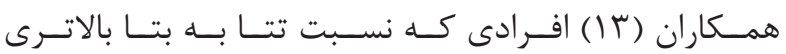

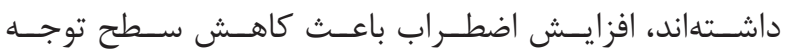

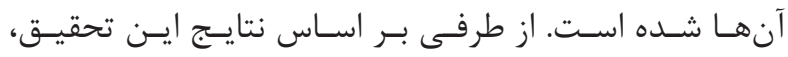

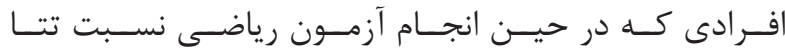

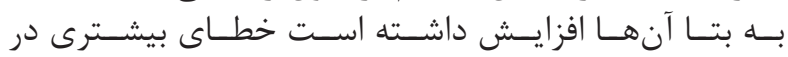

${ }^{10}$ Batashvili 
1. Arghavanian Z, Khanjani Z, Poursharifi $H$. Relationship between Tabriz elementary students' mothers' personality disorders and antisocial behavioral disorder of children in 2008-2009. Procedia-Social and Behavioral Sciences. 2010; 9: 1955-9.

2. Jahanian Najafabadi ASM, Rahmani M, Rahmani M, Imani $\mathrm{H}$. The effect of neurofeedback training on reduce of anxiety. J Res Behave Sci. 2014; 11(6): 657-64.

3. Kaplan HI, Sadock BJ, Grebb JA. Kaplan and Sadock's synopsis of psychiatry: Behavioral sciences, clinical psychiatry. 11th ed wolters kluwer: Williams \& Wilkins Co. 2007; p. 387-418.

4. Hashemi Fesharaki M, Shahgholian N, Kashani F. Effect of stress inoculation training on the levels of stress, anxiety, and depression in hemodialysis patients. Urmia Nursing and Midwifery Faculty. 2016; 14(1): 88-99.

5. Hong E. Test anxiety, perceived test difficulty, and testperformance: Temporal patterns of their effects. Learn Individ Differ. 1999; 11(4): 431-47.

6. Arefi M, Momeni K, Mohsenzadeh F. The effect of cognitive and relaxation therapy on student's test anxiety. Journal of Kermanshah University Medical Sciences. 2012; 16(2): 125-31.

7. Cassady JC, Johnson RE. Cognitive test anxiety and academic performance. Contemporary Educational Psychology. 2002; 27(2): 270-95.

8. Morris RJ, Mather N. Evidence-based interventions for students with learning and behavioral challenges. New York: Routledge. 2008; p. 83-95.

9. King NJ, Ollendick TH, Gullone E. School-related fears of children and adolescents. Aust J educ. 1990; 34(1): 99-112.

10. Najafian J, Akhavan TA. Comparison of the effect of relaxation and biofeedback-assisted relaxation on stress and anxiety score in patients with mild hypertension. Jahrom Medical Journal. 2011; 9: 8-12.

11. Öst L-G, Breitholtz E. Applied relaxation vs. cognitive therapy in the treatment of generalized anxiety disorder. Behav Res Ther. 2000; 38(8): 777-90.

12. Amiri Majd M, Shahmoradi A. The effect of cognitive-behavioral therapy on reducing students test anxiety behavioral sciences. 2009; 1(1): 53-64.

13. Putman P, Verkuil B, Arias-Garcia E, Pantazi I, van Schie C. EEG theta/beta ratio as a potential biomarker for attentional control and resilience against deleterious effects of stress on attention. Cogn Affect Behav Neurosci. 2014; 14(2): 782-91.

14. Demerdzieva A, Pop-Jordanova N. Relation between frontal alpha asymmetry and anxiety in young patients with generalized anxiety disorder. Pril (Makedon Akad Nauk Umet Odd Med Nauki). 2015; 36(2): 157-77.

15. Klados MA, Paraskevopoulos E, Pandria N, Bamidis PD. The impact of math anxiety on working memory: A cortical activations and cortical functional connectivity EEG study. IEEE Access. 2019; 7: 15027-39.

16. Batashvili M, Staples PA, Baker I, Sheffield D. Exploring the relationship between gamma-band activity and maths anxiety. Cognition and Emotion. 2019; 33(8):1-11.

17. Mahram B. Standardization of spielberger anxiety test in Mashhad. Master of Science Thesis, Allameh Tabataba'i University. 1994.

18. Fathi Ashtiani A. Psychological tests personality and mental health. $14^{\text {th }}$ ed. Besat Publication Institute, Tehran. 2018; p. 352-56.

19. Kessler RC, Adler L, Ames M, Demler O, Faraone $\mathrm{S}$, et al. The world health organization adult adhd selfreport scale (asrs): a short screening scale for use in the general population. Psychol Med. , 2005; 35(2): 245-56.

20. Mokhtari H, Rabiei M, Salimi SH. Psychometric properties of the persian version of adult attentiondeficit/hyperactivity disorder self-report scale. Iranian Journal of Psychiatry \& Clinical Psychology. 2015; 21(3): 244-53. 